Pacific Journal of Mathematics

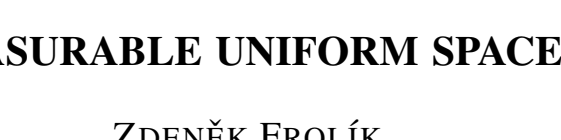




\section{MEASURABLE UNIFORM SPACES}

\section{ZDENĚK FROLÍK}

A uniform space is called $\boldsymbol{\aleph}_{0}$-measurable if the pointwise limit of any sequence of uniformly continuous functions (real valued) is uniformly continuous. A uniform space is called measurable if the pointwise limit of any sequence of uniformly continuous mappings into any metric space is uniformly continuous.

It is shown that measurable spaces are just metric-fine spaces with the property that the cozero sets form a $\sigma$-algebra, or just hereditarily metric-fine spaces.

Metric-fine spaces seem to form a very useful class of spaces; they were introduced by Hager [5], and studied recently by Rice [7] and the author [2], [3]. Separable measurable spaces are studied in Hager [6].

The notation and terminology of Čech [1] is used throughout; for very special terms see Frolík [2]. The main result of the author's [3] is assumed, and [4] may help to understand the motivation.

If $X$ is a uniform space we denote by $\operatorname{coz} X, z X$ or $B a X$ accordingly the cozero sets in $X$ (i.e., the sets $\operatorname{coz} f=\{x \mid f x \neq 0\}$ where $f$ is a uniformly continuous function), or the zero sets in $X$ (i.e., the complements of the cozero sets), or the smallest $\sigma$-algebra which contains $\operatorname{coz} X$ (equivalently: $z X$ ). Since any uniform cover is realized by a mapping into a metric space, the completely coz-additive uniform covers form a basis for the uniformity. Completely coz-additive means that the union of each subfamily is a cozero set.

If $X$ is a uniform space then $e X$ is the set $X$ endowed with the uniformity having the countable uniform covers of $X$ for a basis of uniform covers; $e X$ is a reflection of $X$ in the class of separable uniform spaces (i.e., in spaces $Y$ with $e Y=Y$ ).

We denote by $\alpha$ the usual coreflection into fine uniform spaces. Recall that $\alpha X$ is the set $X$ endowed with the finest uniformity which is topologically equivalent to the uniformity of $X$. The first theorem is a version of a simple classical result on measurable functions. The equivalence of Conditions 1-5 appears in Hager [6]. This theorem is repeatedly used in the sequel, and therefore an economical proof is furnished.

THEOREM 1. Each of the following conditions is necessary and sufficient for a uniform space $X$ to be $\boldsymbol{\aleph}_{0}$-measurable.

1. $e X$ is $\aleph_{0}$-measurable. 
2. $\operatorname{coz} X=z X=B a X$, and every countable partition ranging in $B a X$ is a uniform cover.

3. Each countable partition ranging in $B a X$ is uniform.

4. The countable partitions ranging in BaX form a basis for uniform covers of $e X$.

5. A function $f: X \rightarrow R$ is uniformly continuous if (and only if) the preimages of open sets are the Baire sets in $X$.

Proof. It follows immediately from the definition that Condition 1 is necessary and sufficient. Condition 5 implies that $X$ is $\boldsymbol{N}_{0}$-measurable by the classical result that measurable functions are closed under the operation of taking pointwise limits of sequences ("only if" in Condition 5 is always satisfied). We shall check that each of the Conditions 1-4 implies the subsequent one. Two implications are almost self-evident; namely 2 implies 3 , and for 3 implies 4 we must just recall that $e X$ always has a basis consisting of countable covers ranging in $\operatorname{coz} X(B a X)$.

Condition 4 implies Condition 5, because if $f$ is Baire measurable, and if $\mathscr{U}$ is any countable open cover of $R$, then $f^{-1}[\mathscr{C}]$ is refined by a countable partition ranging in $B a X$.

It remains to show that Condition 1 implies Condition 2. Assume 1. If $G$ is a cozero set, and if $f \geqq 0$ is a uniformly continuous function with $G=\operatorname{coz} f$, then the characteristic (=indicator) function $g$ of $G$ is a pointwise limit of the uniformly continuous functions

$$
f_{n}=\min (1, m \cdot f),
$$

and hence $g$ is uniformly continuous by 1 . Hence $\operatorname{coz} X=z X$, and hence $\operatorname{coz} X$ is a $\sigma$-algebra, and hence $\operatorname{coz} X=B a X$. Now let $\left\{B_{n}\right\}$ be a partition ranging in $B a X$. Let $f_{n}$ be the $n$ multiple of the characteristic function of $B_{n}$. The limit $g$ of uniformly continuous functions $\sum\left\{f_{n} \mid n \leqq k\right\}$ realizes $\left\{B_{n}\right\}$ in the sense that $\left\{B_{n}\right\}=g^{-1}[U]$ for some uniform cover $U$ of $R$. This concludes the proof.

THEOREM 2. For each uniform space $X$ let $M_{\aleph_{0}} X$ be the underlying set of $X$ endowed with the uniformity having for a basis of uniform covers the covers of the following form:

$$
\left\{B_{n} \cap U_{a} \mid n \in N, a \in A\right\}
$$

where $\left\{U_{a}\right\}$ is a uniform cover of $X$, and $\left\{B_{n}\right\}$ is a partition of $X$ ranging in $B a X$.

Then:

1. $e M_{\aleph_{0}} X$ has for a basis of uniform covers the countable partitions ranging in $B a X$. 
2. $M_{\aleph_{0}} X$ is the meet of $X$ and $M_{\aleph_{0}} e X$. spaces.

3. $M_{\aleph_{0}} X$ is a coreflection of $X$ in the category of $\boldsymbol{\aleph}_{0}$-measurable

Proof. 1. The partitions $\left\{B_{n}\right\}$ are uniform because the cover $\left(^{*}\right)$ refines $\left\{B_{n}\right\}$. If $\left\{V_{k}\right\}$ is a countable uniform cover of $M_{\aleph_{0}} X$, take a cover of the form $\left(^{*}\right)$ which refines $\left\{V_{k}\right\}$; we may and shall assume that the union of any subfamily of $\left\{U_{a}\right\}$ belongs to $\operatorname{coz} X$. Put

$$
\begin{aligned}
C_{k n} & =\bigcup\left\{B_{n} \cap U_{a} \mid B_{n} \cap U_{a} \subset V_{k}\right\} \\
& =B_{n} \cap \bigcup\left\{U_{a} \mid B_{n} \cap U_{a} \subset V_{k}\right\} .
\end{aligned}
$$

Clearly $\left\{C_{k n}\right\}$ is a countable cover which ranges in $B a X$ and refines $\left\{V_{k}\right\}$. Now take any partition which refines $\left\{C_{n k}\right\}$. This concludes the proof of 1 .

2. The assertion 2 follows from 1 .

3. Every $M_{\aleph_{0}} X$ is $\aleph_{0}$-measurable by Theorem 1 because obviously

$$
B a M_{\aleph_{0}} X=\operatorname{coz} M_{\aleph_{0}} X=B a X .
$$

Let $f$ be a uniformly continuous mapping of an $\boldsymbol{\aleph}_{0}$-measurable space $Y$ into $X$. We must show that the mapping $f: Y \rightarrow M_{\aleph_{0}} X$ is uniformly continuous. Taking in account the description of $M^{\star}{ }_{0} X$, it is enough to show that the preimage under $f$ of any partition $\left\{B_{n}\right\}$ ranging in $B a X$ is a uniform cover of $Y$, and this follows from Theorem 1 because $f: Y \rightarrow X$ is self-evidently "Baire measurable".

THEOREM 3. The sums, quotients and subspaces of $\boldsymbol{\aleph}_{0}$-measurable spaces are $\aleph_{0}$-measurable.

Proof. This follows immediately from Theorem 1.

REMARK. Theorem 3 implies by a purely categorical argument that $\boldsymbol{W}_{0}$-measurable spaces form a coreflective category, and also the coreflectivity of $\boldsymbol{\aleph}_{0}$-measurable spaces (established in Theorem 2) implies that the sums and the quotients of $\boldsymbol{\aleph}_{0}$-measurable spaces are $\boldsymbol{\aleph}_{0}{ }^{-}$ measurable, again by a purely categorial argument.

For separable uniform spaces the next theorem is Hager [6, 6.5].

THEOREM 4. Each of the following two conditions is necessary and sufficient for a uniform space $X$ to be $\boldsymbol{\aleph}_{0}$-measurable:

(1) Every uniformly continuous function on $X$ factorizes through $M_{\aleph_{0}} R$.

(2) Every uniformly continuous mapping of $X$ into a separable metrizable space $S$ factorizes through $M_{\aleph_{0}} S$. 
Proof. Since $M_{\aleph_{0}}$ is a functor Condition (2) is necessary, and clearly (2) implies (1). Condition (1) implies immediately that the pointwise limit of uniformly continuous functions is uniformly continuous.

For the next result we need to recall further definitions. A uniform space $X$ is called metric-fine if for every uniformly continuous mapping $f$ of $X$ into a metric space $M$ the mapping $f: X \rightarrow \alpha M$ (see introduction) is uniformly continuous. A uniform space is called (separable metric)-fine if the condition is fulfilled for $f$ 's into separable $M$ 's. For properties of metric-fine and (separable metric)-fine spaces we refer to Frolík [3]; Hager [5] is a good reference, but it is not enough for our purpose. We need the following description of the coreflections $m_{\boldsymbol{N}_{0}} X$ and $m X$ of a uniform space $X$ in (separable metric)fine or metric-fine spaces respectively (see Frolík [3, Theorems 1 and 3]:

The covers of the form

$$
\left\{U_{a} \cap B_{n} \mid a \in A, n \in N\right\}
$$

form a basis for $m_{\aleph_{0}} X$, and the covers

$$
\left\{U_{a}^{n} \cap B_{n} \mid n \in N, a \in A_{n}\right\}
$$

form a basis for the uniform covers of $m X$, where $\left\{U_{a} \mid a \in A\right\}$, $\left\{U_{a}^{n} \mid a \in A_{n}\right\}$ are uniform covers of $X$, and $\left\{B_{n}\right\}$ is a cover of $X$ by elements of $\operatorname{coz} X$; in addition we may assume that all covers are completely $\operatorname{coz} X$-additive.

We also need to know that

$$
e m X=e m_{\aleph_{0}} X=m e X=m_{\aleph_{0}} e X .
$$

A uniform space $X$ is called inversion-closed if the set $U(X)$ of all uniformly continuous functions is inversion-closed, and this means, that if $f \in U(X)$ and $f x \neq 0$ for all $x \in X$, then $1 / f$ is uniformly continuous.

If $X$ is (separable metric)-fine then $X$ is inversion-closed; this is obvious.

LEMMA 1. Let $Y$ be an inversion-closed subspace of a uniform space $X$. For each zero set $Z \subset X-Y$ there exists a zero set $Z^{\prime} \supset Y$ such that $Z^{\prime} \cap Z=\phi$. Hence, if $Y$ is a cozero set in $X$, then $Y$ is a zero set.

Proof. Take a nonnegative function $f$ in $U(X)$ such that $Z=$ $\{x \mid f x=0\}$, and let $g$ be the inversion of the restriction of $f$ to $Y$. Take a uniformly continuous pseudometric $d$ on $Y$ such that $f$ is uniformly continuous on $\langle X, d\rangle$, and $g$ is uniformly continuous on the 
subspace $Y$ of $\langle X, d\rangle$. The function $g$ extends to a uniformly continuous function $g^{\prime}$ on the closure $Z^{\prime}$ of $X$ in $\langle X, d\rangle ; Z^{\prime}$ is a zero set in $\langle X, d\rangle$, hence in $X$. We shall check that $Z^{\prime} \cap Z=\phi$; if $z \in Z^{\prime} \cap Z$, then $f z=0$, and a sequence $\left\{y_{n}\right\}$ in $Y$ converges to $z$; in $\langle X, d\rangle$, since $f z=0$ necessarily $f y_{n} \rightarrow 0$; hence the value of the extended $g$ should be $\infty \notin R$, and this contradiction proves the lemma.

REMARK. In the proof of Lemma 1 we used the following simple but useful proposition:

If $Y \subset X, M$ is metric, and $g: Y \rightarrow M$ is uniformly continuous, then there is a uniformly continuous pseudometric $d$ on $X(X !)$ such that $g$ is uniformly continuous on $\langle Y, d\rangle$. (Proof. For each $n$, let $u_{n}$ be a uniform cover of $X$ such that the trace of $u_{n}$ on $Y$ refines the inverse image under $g$ of the $1 / n$-cover of $M$. Arrange it so that $u_{n+1}$ star-refines $u_{n}$ for each $n$, and let $d$ be the pseudometric associated with the sequence $\left\{u_{n}\right\}$.) The existence of the $d$ in the proof of Lemma 1 now follows. We note that the proposition implies that if $Y \subset X$ and $g: Y \rightarrow R$ is uniformly continuous, then $g$ has a continuous extension over $X$ : Choose $d$ as above, extend $g$ over the $d$-closure of $Y$ by uniform continuity, then over all a $X$ by the Tietze-Urysohn Theorem. (If $g$ is bounded, there is a uniformly continuous extension by Katětov's well known theorem.)

THEOREM 5. The following properties of a uniform space $X$ are equivalent:

1. $X$ is $\boldsymbol{\aleph}_{0}$-measurable.

2. $X$ is hereditarily (separable metric)-fine.

3. $X$ is (separable metric)-fine, and each subspace is inversionclosed.

4. $X$ is (separable metric)-fine, and each cozero subspace of $X$ is inversion-closed.

Proof. Since $\boldsymbol{Y}_{0}$-measurable is hereditary and implies (separable metric)-fine, Condition 1 implies Condition 2. Next (separable metric)fine implies inversion-closed, and hence Condition 2 implies Condition 3. Self-evidently Condition 3 implies Condition 4. It remains to show that Condition 4 implies Condition 1. Assume 4. By Lemma 1 we get $\operatorname{coz} X=z X$, hence $\operatorname{coz} X=B a X$. As is noted above, since $X$ is (separable metric)-fine, this implies that $X$ is $\boldsymbol{Y}_{a_{0}}$-measurable.

REMARK. For separable spaces, the equivalence of 1 and 2 in Theorem 5 is in Hager [5, 4.2]. We are in a good position to derive several results which are not needed in the sequel, but may help the reader to get better understanding of the spaces used. Again for separable 
spaces, Propositions 1, 2, 3 and the corollaries appear in Hager [5].

Proposition 1. The following properties of a subspace $Y$ of a (separable metric)-fine space $X$ are equivalent:

1. $Y$ is inversion-closed.

2. $Y$ is (separable metric)-fine.

3. If $G \supset Y$ is a cozero set, then $Y \subset Z \subset G$ for some zero set $Z$.

Proof. By Lemma 1 Condition 1 implies Condition 3, and obviously Condition 2 implies Condition 1. The remaining implication is obtained as follows: If $\left\{U_{n}\right\}$ is a countable cover of $Y$ by cozero sets in $Y$, then we can take cozero sets $G_{n}$ in $X$ such that $G_{n} \cap Y=U_{n}$, and apply Lemma 1 to $Y$, the complement $Z$ of $\bigcup\left\{G_{n}\right\}$, and to $X$. Let $G^{\prime}$ be the complement of $Z^{\prime}$. Clearly all $G_{n}$ together with $G^{\prime}$ form a countable cover of $X$, which consists of cozero sets in $X$, hence form a uniform cover of $X$. The $\left\{U_{n}\right\}$ is just the trace of the cover on $Y$.

Corollary. If $Y \subset X$, then $m_{\aleph_{0}} Y$ is a subspace of $m_{\aleph_{0}} X$ if and only if Condition 3 of Proposition 1 holds.

The following Proposition 2 is a corollary to Corollary.

Proposition 2. Let $Y$ be a dense subspace of a uniform space $X$. Then $m_{\aleph_{0}} Y$ is a subspace of $m_{\aleph_{0}} X$ if and only if $Y$ is $G_{\delta}$-dense in $X$ (i.e., $X-Y$ contains no nonvoid $G_{i}$-set, or equivalently, no nonvoid zero set).

Finally:

Proposition 3. Let $K$ be a compactification of a topological space $X$ (completely regular). The following properties are equivalent:

1. $K$ is the Samuel compactification of some metric-fine uniformity on $X$.

2. $K$ is the Samuel compactification of some inversion-closed uniformity on $X$.

3. If $G$ is a cozero set in $K, X \subset G \subset K$, then $K$ is a Čech-Stone compactification of $G$.

Proof. Since every metric-fine uniformity is inversion-closed, Condition 1 implies Condition 2. Assume Condition 2, and let $g$ be a bounded continuous function on $G \supset X, G$ being a cozero set in $K$. Pick up a bounded nonnegative continuous function $f$ on $K$ such that $G=\operatorname{coz} f$. The function $f \cdot g$ on $G$ extends to a continuous function $h$ on $K$; indeed, put $h x=0$ for $x$ in $K-G$. Thus the restriction of $g$ 
to $X$ is the ration of two uniformly continuous functions, namely

$$
g x=h x / f x,
$$

hence is uniformly continuous, and hence extends to $K$.

Assume Condition 3, and let us consider the (separable metric)-fine coreflection of the relativization of the uniformity of $K$ to $X$. We must show that every uniformly continuous bounded function $f$ extends to $K$, and in view of Condition 3 , it is enough to extend $f$ to a cozero set $G \supset X$. Take a countable base $\left\{U_{n}\right\}$ for $R$ and extend each $U_{n}$ to a cozero set $G_{n}$ in $R$; let $G$ be the union of all $G_{n}$. Clearly $f$ is uniformly continuous with respect to the relativization of the fine uniformity of $G$ to $X$, and hence $f$ extends to a continuous function on $G$. This completes the proof.

CoRollary. The Samuel compactification of a uniform space $X$ enjoys the properties in Proposition 3 if and only if $m X$ is proximally equivalent to $X$.

For more results on rings of uniformly continuous functions we refer to Hager [5].

Now we proceed to measurable spaces which seem to be quite interesting. The first result is a characterization of measurable spaces which will be used to describe the coreflection into measurable spaces, and which connects immediately the theory of measurable spaces with the theory of metric-fine spaces.

THeOREM 6. A uniform space $X$ is measurable if and only if for any sequence $\left\{\left\{U_{a}^{n} \mid a \in A_{n}\right\}\right\}$ of uniform covers of $X$, and for any partition $\left\{B_{n}\right\}$ of $X$ ranging in $B a X$ the cover

$$
\left\{B_{n} \cap U_{a}^{n} \mid n \in N, a \in A_{n}\right\}
$$

is uniform.

Proof. First assume that $X$ is measurable, and let $\left(^{*}\right)$ be given. We shall realize $\left(^{*}\right)$ by a uniformly continuous mapping $g$ into a metric space $Y$.

Since $X$ is $\boldsymbol{\aleph}_{0}$-measurable, for each $n$ the cover

$$
\mathscr{V}_{n}=\left\{B_{k} \cap U_{a}^{n} \mid k \in N, a \in A_{n}\right\}
$$

is uniform, and hence there exists a uniformly continuous mapping $f$ of $X$ into a metric space $\langle M, d\rangle$, which realizes all $\mathscr{V}_{n}$. We may and shall assume that $d \leqq 1$, and the preimage of the $1 / n$-cover of 
$\langle M, d\rangle$ under $f$ refines $\mathscr{V}_{k}$ for $k \leqq n$. In particular, the preimage of the 1-cover of $M, d$ refines $\left\{B_{k}\right\}$. Hence $C_{k}=f\left[B_{k}\right]$ form a uniformly discrete partition of $\langle M, d\rangle$. Now let $Y$ be the set $N \times M$ endowed with a metric $D$ defined as follows:

$$
\begin{aligned}
D\langle\langle n, y\rangle,\langle m, z\rangle\rangle & =1 \text { if } n \neq m, \\
& =\min (1, n . d\langle y, z\rangle) \text { if } n=m .
\end{aligned}
$$

If we put $d_{n}=\min (1, n . d)$, then $d_{n}$ is a metric for $M$ uniformly equivalent to $d$, and

$$
J_{n}=\{y \longrightarrow\langle n, y\rangle\}:\left\langle M, d_{n}\right\rangle \longrightarrow\langle Y, D\rangle
$$

is metric preserving (hence uniform embedding).

Define a sequence $\left\{h_{n}\right\}$ of uniformly continuous mappings of $M$ into $Y$, and a mapping $h: M \rightarrow Y$ (which will not be uniformly continuous in general) as follows:

$$
\begin{aligned}
g y & =\langle n, y\rangle \text { for } y \text { in } C_{n}, \\
g_{n} y & =\langle k, y\rangle \text { for } y \text { in } C_{k} \text { with } k \leqq n, \\
& =\langle n, y\rangle \text { for } y \text { in } C_{k} \text { with } k \geqq n .
\end{aligned}
$$

The mappings $g_{n}: M \rightarrow Y$ are uniformly continuous, because

$$
\begin{aligned}
& g_{n}=J_{k} \quad \text { on } \quad B_{k} \text { with } k<n \\
& g_{n}=J_{n} \text { on } \quad \bigcup\left\{B_{k} \mid k \geqq n\right\} .
\end{aligned}
$$

For each $y$ in $M$ the sequence $\left\{g_{n} y\right\}$ is eventually constant and converges to $g y$, namely if $y \in C_{k}$ then $g_{n} y=g y$ for $n \geqq k$.

Now let $h=g \circ f, h_{n}=g_{n} \circ f$. The mappings $h_{n}$ are uniformly continuous, and hence $h$ is uniformly continuous because $\left\{h_{n}\right\}$ converges pointwise to $h$ and $X$ is measurable.

It is easy to check that the preimage of the 1-cover $\mathscr{U}$ of $Y$ under $h$ refines our given cover $(*)$. Indeed,

$$
h^{-1}[n \times M]=f^{-1}\left[C_{n}\right]=B_{n},
$$

and if $U$ is the open sphere of radius 1 centered at a point $\langle n, y\rangle$, then $U \subset n \times M$ and $V=J_{n}^{-1}[U]$ is the open sphere of radius 1 in $\left\langle M, d_{n}\right\rangle$ centered at $y$, and hence $V$ is the sphere of radius $1 / n$ in $\langle M, d\rangle$ centered at $y$, and hence $f^{-1}[V]$ is contained in some $U_{a}^{n}$. Thus

$$
h^{-1}[U]=f^{-1}\left[J_{n}^{-1}[U]\right]
$$

is contained in $U_{a}^{n}$, and since $U \subset n \times M$,

$$
h^{-1}[U] \subset B_{n} \cap U_{a}^{n} .
$$

This concludes the proof. 
Now assume the condition, and let $\left\{f_{n}\right\}$ be a sequence of uniformly continuous mappings of $X$ into a metric space $M$, which pointwise converges to a mapping $f: X \rightarrow M$. We must show that $f: X \rightarrow M$ is uniformly continuous. For each positive number $r$, and for each $n$ consider the set

$$
B_{n}^{r}=\left\{x \mid d\left\langle f_{k} x, f_{l} x\right\rangle \leqq r \text { for } k, h \geqq n\right\} .
$$

Thus $d\left\langle f x, f_{l} x\right\rangle \leqq r$ for $x \in B_{n}^{r}, l \geqq n$. Clearly the union of the sequence $\left\{B_{n}^{r}\right\}$ is $X$ for each $r$, and each $B_{n}^{r}$ belongs to $B a X$. Now given any positive number $\varepsilon$ choose a uniform cover $\left\{U_{a}^{n} \mid a \in A_{n}\right\}$ such that the diameter of $f_{n}\left[U_{a}^{n}\right]$ is less than $1 / 3 \cdot \varepsilon$ for each $a$ in $A_{n}$. Finally put

$$
B_{n}=B_{n}^{r}-B_{n-1}^{r}
$$

with $r=1 / 3 \varepsilon$. Clearly the diameter of each $f\left[B_{n} \cap U_{a}^{n}\right]$ is at most $\varepsilon$. By our assumption $\left\{B_{n} \cap U_{a}^{n}\right\}$ is a uniform cover, and hence $f$ is uniformly continuous. This concludes the proof.

THEOREM 7. The sums, subspaces and quotients of measurable spaces are measurable.

Proof. By a routine argument from Theorem 6.

THEOREM 8. The following conditions on a uniform space $X$ are equivalent:

1. $X$ is measurable.

2. $X$ is $\boldsymbol{\aleph}_{0}$-measurable and metric-fine.

3. $X$ is hereditarily (separable-metric)-fine and metric-fine.

4. $X$ is hereditarily metric-fine (i.e., each subspace of $X$ is metric-fine).

Proof. If we compare the characterization of metric-fine spaces recalled above and Theorem 6 we see that Conditions 1 and 2 are equivalent. Conditions 2 and 3 are equivalent by Theorem 5. Finally, obviously Condition 4 implies Condition 3, and is implied by Condition 1 because measurable spaces are hereditary.

It follows from Theorem 7 that measurable spaces are coreflective. Now we shall describe a coreflection measurable spaces and get as $a$ byproduct that measurable spaces are coreflective.

THEOREM 8. For every uniform space $X$ let $M X$ be the set $X$ endowed with the uniformity having for a basis of uniform covers the covers of the form described in Theorem 6. Then:

1. eMX has for a basis of uniform covers the countable partitions ranging in $B a X$, and hence $e M X$ is $\boldsymbol{\aleph}_{0}$-measurable, and $B a X=$ 
$B a M X$.

2. $e M X=e M_{\aleph_{0}} X=M e X=M_{\aleph_{0}} e X$.

3. $M X$ is a coreflection of $X$ in measurable spaces.

Proof. Let $\left\{W_{k}\right\}$ be a countable cover of $M X$, and let

$$
\left\{U_{a}^{n} \cap B_{n} \mid n \in N, a \in A_{n}\right\}
$$

be a defining cover which refines $\left\{W_{k}\right\}$. We may and shall assume that $\left\{U_{a}^{n} \mid a \in A_{n}\right\}$ are completely coz-additive (such covers form a basis for every uniform space). Put

$$
\begin{aligned}
C_{k n} & =\bigcup\left\{U_{a}^{n} \cap B_{n} \mid U_{a}^{n} \cap B_{n} \subset W_{k}\right\} \\
& =B_{n} \cap \bigcup\left\{U_{a}^{n} \mid U_{a}^{n} \cap B_{n} \subset W_{k}\right\} .
\end{aligned}
$$

It is easily seen that $\left\{C_{k n}\right\}$ is a countable cover which ranges in $B a X$, and $\left\{C_{k n}\right\}$ refines $\left\{W_{k}\right\}$. Thus the countable partitions ranging in $B a X$ form a basis for uniform covers of $e M X$, hence $B a X=\operatorname{coz} M X=$ $B a M X$, hence $e M X$ is $\aleph_{0}$-measurable. This proves 1 .

It follows from 1 and Theorem 2 that $e M X=e M_{\aleph_{0}} X$, again by Theorem 2 we have $e M_{\aleph_{0}} X=M_{\aleph_{0}} e M$. If $X$ is separable then clearly $M X$ is separable (we may take all $\left\{U_{a}^{n}\right\}$ in the basis consisting of countable uniform covers, and then the defining covers are countable), and hence $M_{\aleph_{0}} e X=M e X$. This concludes the proof of 2 .

Every space $M X$ is measurable, because it follows from the definition of $M X$ and from 1 that $M M X=M X$, and by Theorem $6 X$ is measurable if and only if $M X=X$. It remains to show that if $f: Z \rightarrow X$ is uniformly continuous and if $Z$ is measurable then $f: Z \rightarrow$ $M X$ are measurable. This follows from Theorem 6, and the definition of $M X$. This concludes the proof.

The next result says that the functor $M$ is metrically determined.

THeORem 9. MX is projectively generated by mappings $f: M X \rightarrow$ $M P$ where $f$ are uniformly continuous mappings of $X$ into metric spaces $P$. A uniform space $X$ is measurable if and only if for each uniformly continuous mapping $f$ of $X$ into a metric space $P$ the mapping $f: X \rightarrow M P$ is uniformly continuous.

Proof. The second assertion follows immediately from the first one. The first assertion follows from Theorem 8, because any sequence of uniform covers, and a sequence of Baire sets may be realized in a metric space by a uniformly continuous mapping. To be sure we formulate the fact about the realization of Baire sets in a lemma.

Lemma 2. Let $\left\{B_{n}\right\}$ be a sequence of Baire sets in a uniform 
space $X$. Then there exists a uniformly continuous mapping $f$ into a separable metric space $S$, and a sequence $\left\{C_{n}\right\}$ of Baire sets in $S$ such that $f^{-1}\left[C_{n}\right]=B_{n}$ for each $n$.

Proof. Take a countable collection $\left\{U_{a} \mid a \in A\right\}$ of cozero sets in $X$ such that all $B_{n}$ belong to the smallest $\sigma$-algebra containing all $U_{a}$. We may and shall assume that $A=N$. Take uniformly continuous functions $f_{n}$ such that

$$
U_{n}=\operatorname{coz} f_{n},
$$

and $0 \leqq f_{n} \leqq 1 / 2^{n}$. Then $f_{n}$ are uniformly continuous, and

$$
f: X \longrightarrow R^{N}
$$

has the required properties, where $f$ is the reduced product of $\left\{f_{n}\right\}$, i.e., $f x=\left\{f_{n} x\right\}$. This concludes the proof.

The next result describes a nice basis for $M X$.

THEOREM 10. The space $M X$ has for a basis of uniform covers the collection of all $\sigma$-uniformly discrete (in $X$ ) partitions of bounded class in BaX.

Corollary. A space $X$ is measurable if and only if each $\sigma$ uniformly discrete partition of bounded class in BaX is a uniform cover of $X$.

We must explain the notion "of bounded class in $B a X$ ". We know that $B a X$ is the smallest $\sigma$-algebra which contains $\operatorname{coz} X$ (or equivalently, $z X)$. It follows that

$$
\begin{aligned}
B a X & =\bigcup\left\{\mathscr{B}_{\alpha} \mid \alpha<\omega_{1}\right\} \\
& =\bigcup\left\{\mathscr{B}_{\alpha}^{1} \mid \alpha<\omega_{1}\right\}
\end{aligned}
$$

where $\mathscr{B}_{0}=\operatorname{coz} X, \mathscr{B}_{0}^{1}=z X$, and by induction $\mathscr{B}_{\alpha}\left(\mathscr{B}_{\alpha}^{1}\right.$, resp.) is obtained from $\bigcup\left\{\mathscr{B}_{\beta} \mid \beta<\alpha\right\}\left(\bigcup\left\{\mathscr{B}_{\beta}{ }^{1} \mid \beta<\alpha\right\}\right)$ by taking all countable intersections (countable unions) or countable unions (countable intersections) according to as $\alpha$ is odd or even.

Definition. A family $\left\{X_{a}\right\}$ is of bounded class in $B a X$ if $\left\{X_{a}\right\}$ ranges in some $\mathscr{B}_{\alpha} \cup \mathscr{B}_{\alpha}^{1}$; the smallest $\alpha$ is called the class of $\left\{X_{a}\right\}$.

Proof of Theorem 10. Let $\left\{X_{a}^{n} \mid n \in N, a \in A\right\}$ be a $\sigma$-discrete partition of bounded class, say $\alpha$, in $B a X$. Put $B_{n}=\bigcup\left\{X_{a}^{n} \mid a \in A_{n}\right\}$. The sets $B_{n}$ are of class at most $\alpha+1$ because $\left\{X_{a}^{n} \mid a \in A_{n}\right\}$ are uniformly discrete. The sets $X_{a}^{n}$ are cozero sets in $B_{n}$, and they form 
a uniform cover of the subspace $B_{n}$ of $X$. By Theorem $6,\left\{X_{a}^{n}\right\}$ is a uniform cover of $M X$.

It remains to show that these covers form a basis. By A. H. Stone Theorem every uniform cover $\mathscr{C}$ of every uniform space $X$ has a uniformly $\sigma$-discrete refinement $\mathscr{V}=\bigcup\left\{V_{k}\right\}$; $\mathscr{V}$ is not necessarily uniform, but it is a uniform cover of $M X$ by Theorem 6 (in fact it is a uniform cover of $m X$, which is the coreflection in metric-fine spaces); indeed put $C_{n}=\bigcup\left\{\mathscr{V}_{n}\right\}, B_{n}=C_{n}-\bigcup\left\{C_{k} \mid k<n\right\}$. Now if $\left\{U_{a}^{n} \cap B_{n}\right\}$ is a typical defining cover of $M X$, we may replace each cover $\left\{U_{a}^{n} \mid a \in A\right\}$ by a uniformly (in $X$ ) $\sigma$-discrete cover $\left\{V_{k}^{n} \mid k \in N\right\}$, and put $B_{n k}=B_{n} \cap V_{n}^{k}$. Then $\bigcup\left\{B_{n k} \cap\left[\mathscr{V}_{k}^{n}\right]\right\}$ is a uniformly (in $X$ ) $\sigma$ discrete cover of a bounded class which refines $\left\{U_{a}^{n} \cap B_{n}\right\}$. We need a partition; well order $\{(n, k)\}$ according to $\omega_{0}$, and take the differences as above. This concludes the proof.

In conclusion we show that for mappings of metric-fine (and hence of measurable) spaces uniform continuity depends on two data only: Cozero sets and " $\sigma$-discreteness". I do not know whether this property characterizes metric-fine spaces. Recall (we shall not use it) that just metric-fine proximally fine spaces are completely determined by cozero sets, see Frolik [3, Theorem 4]. First let us stress that the only distinction between metric-fine spaces and measurable ones is in cozero sets.

THEOREM 11. A uniform space $X$ is measurable if and only if $\operatorname{coz} X=B a X$, and $X$ is metric-fine.

Proof. This follows immediately from Theorems 1 and 7.

Theorem 12. Assume that $X$ is metric-fine. A mapping $f$ of $X$ into a uniform space $Y$ is uniformly continuous if (and obviously, only if) it enjoys the following properties:

A. The preimages of cozero sets are cozero sets.

B. The preimages of uniformly $\sigma$-discrete families are uniformly $\sigma$-discrete.

Proof. Assume that $X$ is metric-fine, and that $f: X \rightarrow Y$ satisfies Conditions $\mathrm{A}$ and $\mathrm{B}$. To prove that $f: X \rightarrow Y$ is uniformly continuous it is enough to show that

$$
h=g \circ f: X \longrightarrow Z
$$

is uniformly continuous for every uniformly continuous mapping $g$ of $Y$ into a metrizable space $Z$. If $\mathscr{C}$ is any uniform cover of $Z$, then by the A.H. Stone Theorem we can take a uniformly $\sigma$-discrete open 
refinement $\mathscr{V}=\bigcup\left\{\mathscr{V}_{n}\right\}$ (not necessarily uniform), and the preimage of $\mathscr{V}$ under $h$ is, in view of Conditions $\mathrm{A}$ and $\mathrm{B}$, uniform by Theorem 2 in Frolík [3], which was recalled just after Theorem 5.

REMARK. M. Rice [7] proved independently that a space $X$ is hereditarily metric-fine if and only if the condition in Theorem 6 is satisfied.

\section{REFERENCES}

1. E. Čech, Topological Spaces, Academia, Prague, 1965.

2. Z. Frolík, Interplay of measurable and uniform spaces, Proc. 2nd International Topological Conference in Yugoslavia, Budva, 1972.

3. — A note on metric-fine spaces, Proc. Amer. Math. Soc.

4. - Topological methods in measure theory and measurable spaces, Proc. 3rd Prague Symposium in Topology and Applications, Prague, 1971. Academia (Academic Press), Prague, 1972.

5. A. Hager, Some nearly fine uniform spaces, Proc. London Math. Soc.

6. - Measurable uniform spaces, Fund. Math., 77 (1972), 51-73.

7. M. Rice, Thesis, Wesleyan University, 1973.

8. Covering and function theoretic properties of uniform spaces, Bull. Amer. Math. Soc.

9. J. Isbell, Uniform spaces, Math. Surveys 12, Amer. Math. Soc., 1964.

10. A. H. Stone, Paracompactness and product spaces, Bull. Amer. Math. Soc., 54 (1948), 977-982.

Received March 2, 1973.

Mathematics Institute of ČSAV

AND

University of Pittsburgh 



\section{PACIFIC JOURNAL OF MATHEMATICS}

EDITORS

RICHARD ARENS (Managing Editor)

University of California

Los Angeles, Calıfornia 90024

R. A. Beaumont

University of Washington

Seattle, Washington 98105
J. DugundJI

Department of Mathematics

University of Southern California

Los Angeles, California 90007

D. Gilbarg and J. Milgram

Stanford University

Stanford, California 94305

\section{ASSOCIATE EDITORS}
E. F. BECKENBACH
B. H. NeumanN
F. WOLF
K. YOSHIDA

\section{SUPPORTING INSTITUTIONS}

UNIVERSITY OF BRITISH COLUMBIA

CALIFORNIA INSTITUTE OF TECHNOLOGY

UNIVERSITY OF CALIFORNIA

MONTANA STATE UNIVERSITY

UNIVERSITY OF NEVADA

NEW MEXICO STATE UNIVERSITY

OREGON STATE UNIVERSITY

UNIVERSITY OF OREGON

OSAKA UNIVERSITY
UNIVERSITY OF SOUTHERN CALIFORNIA

STANFORD UNIVERSITY

UNIVERSITY OF TOKYO

UNIVERSITY OF UTAH

WASHINGTON STATE UNIVERSITY

UNIVERSITY OF WASHINGTON

AMERICAN MATHEMATICAL SOCIETY NAVAL WEAPONS CENTER 


\section{Pacific Journal of Mathematics}

\section{Vol. 55, No. $1 \quad$ September, 1974}

Robert Lee Anderson, Continuous spectra of a singular symmetric

differential operator on a Hilbert space of vector-valued functions . . . $\quad 1$

Michael James Cambern, The isometries of $L^{p}(X, K) \ldots \ldots \ldots \ldots \ldots . . \ldots$

R. H. Cameron and David Arne Storvick, Two related integrals over spaces of continuous functions ................................

Gary Theodore Chartrand and Albert David Polimeni, Ramsey theory and

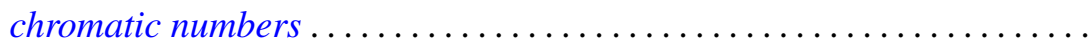

John Deryck De Pree and Harry Scott Klein, Characterization of collectively compact sets of linear operators ...................

John Deryck De Pree and Harry Scott Klein, Semi-groups and collectively compact sets of linear operators ....................... 55

George Epstein and Alfred Horn, Chain based lattices.............. 65

Paul Erdős and Ernst Gabor Straus, On the irrationality of certain series . . 85

Zdeněk Frolík, Measurable uniform spaces................... 93

Stephen Michael Gagola, Jr., Characters fully ramified over a normal

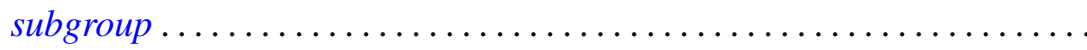

Frank Larkin Gilfeather, Operator valued roots of abelian analytic

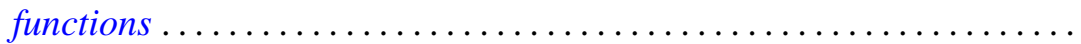

D. S. Goel, A. S. B. Holland, Cyril Nasim and B. N. Sahney, Best approximation by a saturation class of polynomial operators

James Secord Howland, Puiseux series for resonances at an embedded

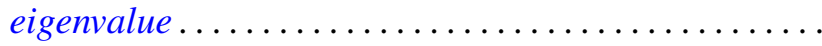

David Jacobson, Linear GCD equations .................

P. H. Karvellas, A note on compact semirings which are multiplicative

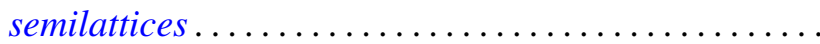

Allan Morton Krall, Stieltjes differential-boundary operators. II . .

D. G. Larman, On the inner aperture and intersections of convex sets

S. N. Mukhopadhyay, On the regularity of the $P^{n}$-integral and its application to summable trigonometric series ....... .

Dwight Webster Read, On $(J, M, m)$-extensions of Boolean algebras ....

David Francis Rearick, Multiplicativity-preserving arithmetic power series.

Indranand Sinha, Characteristic ideals in group algebras

Charles Thomas Tucker, II, Homomorphisms of Riesz spaces . . .

Kunio Yamagata, The exchange property and direct sums of indecomposable injective modules. 